\section{(1)}

CrossMark

\title{
Alcohol consumption as a risk factor for tuberculosis: meta-analyses and burden of disease
}

\author{
Sameer Imtiaz ${ }^{1,2}$, Kevin D. Shield ${ }^{3}$, Michael Roerecke ${ }^{2,4}$, \\ Andriy V. Samokhvalov ${ }^{1,2,5}$, Knut Lönnroth ${ }^{6,7}$ and Jürgen Rehm², 2,4,5,8,9
}

Affiliations: ${ }^{1}$ Institute of Medical Science, University of Toronto, Toronto, ON, Canada. ${ }^{2}$ Institute for Mental Health Policy Research, Centre for Addiction and Mental Health, Toronto, ON, Canada. ${ }^{3}$ Section of Cancer Surveillance, International Agency for Research on Cancer, Lyon, France. ${ }^{4}$ Dalla Lana School of Public Health, University of Toronto, Toronto, ON, Canada. ${ }^{5}$ Department of Psychiatry, University of Toronto, Toronto, ON, Canada. ${ }^{6}$ Global TB Programme, World Health Organization, Geneva, Switzerland. ${ }^{7}$ Department of Public Health Sciences, Karolinska Institutet, Stockholm, Sweden. ${ }^{8}$ Campbell Family Mental Health Research Institute, Centre for Addiction and Mental Health, Toronto, ON, Canada. ${ }^{9}$ Institute for Clinical Psychology and Psychotherapy, Technische Universität Dresden, Dresden, Germany.

Correspondence: Sameer Imtiaz, Institute for Mental Health Policy Research, Centre for Addiction and Mental Health, T521 - 33 Russell Street, Toronto, Canada, M5S 2S1. E-mail: sameer.imtiazagmail.com

@ERSpublications

Alcohol use, alcohol dosage and alcohol-related problems are associated with an increased risk of tuberculosis http://ow.ly/CheO30aPqJg

Cite this article as: Imtiaz S, Shield KD, Roerecke M, et al. Alcohol consumption as a risk factor for tuberculosis: meta-analyses and burden of disease. Eur Respir J 2017; 50: 1700216 [https://doi.org/10.1183/ 13993003.00216-2017].

ABSTRACT Meta-analyses of alcohol use, alcohol dosage and alcohol-related problems as risk factors for tuberculosis incidence were undertaken. The global alcohol-attributable tuberculosis burden of disease was also re-estimated.

Systematic searches were conducted, reference lists were reviewed and expert consultations were held to identify studies. Cohort and case-control studies were included if there were no temporal violations of exposure and outcome. Risk relations (RRs) were pooled by using categorical and dose-response metaanalyses. The alcohol-attributable tuberculosis burden of disease was estimated by using alcoholattributable fractions.

36 of 1108 studies were included. RRs for alcohol use and alcohol-related problems were 1.35 (95\% CI 1.09-1.68; $\mathrm{I}^{2}:$ 83\%) and 3.33 (95\% CI 2.14-5.19; 87\%), respectively. Concerning alcohol dosage, tuberculosis risk rose as ethanol intake increased, with evidence of a threshold effect. Alcohol consumption caused 22.02 incident cases (95\% CI 19.70-40.77) and 2.35 deaths (95\% CI 2.05-4.79) per 100000 people from tuberculosis in 2014. Alcohol-attributable tuberculosis incidence increased between 2000 and 2014 in most high tuberculosis burden countries, whereas mortality decreased.

Alcohol consumption was associated with an increased risk of tuberculosis in all meta-analyses. It was consequently a major contributor to the tuberculosis burden of disease.

This article has supplementary material available from erj.ersjournals.com

Received: Jan 302017 | Accepted after revision: April 082017

Conflict of interest: Disclosures can be found alongside this article at erj.ersjournals.com

Copyright OERS 2017. This version is distributed under the terms of the Creative Commons Attribution NonCommercial Licence 4.0.

This is one of a selection of articles published as ERJ Open papers, as part of an initiative agreed between the European Respiratory Society and the World Health Organization. 


\section{Introduction}

Although the incidence of tuberculosis has been declining since 2000, 10.4 million incident cases were estimated globally in 2015 [1]. Tuberculosis also caused an estimated 1.4 million deaths globally during the same time frame, surpassing human immunodeficiency virus (HIV) and bolstering its status as a leading cause of death [1]. As such, tuberculosis is a major infectious cause of the global burden of disease; it contributes $1.6 \%$ of all disability-adjusted life years and $2.0 \%$ of all deaths, according to the Global Burden of Disease Study 2015 [2].

An important risk factor for tuberculosis is alcohol consumption, particularly heavy consumption [3, 4]. A meta-analysis conducted by LöNNROTH et al. [3], which included studies published up to 2007, indicated that alcohol consumption of more than $40 \mathrm{~g}$ of ethanol per day or a diagnosis of an alcohol use disorder resulted in a nearly three-fold increase in the risk of tuberculosis. Based on this meta-analysis, alcohol consumption was estimated to be responsible for approximately $10 \%$ of all incident cases and deaths due to tuberculosis [4]. Since then a number of relevant studies have been published, which allow separate meta-analyses to be conducted of alcohol use, alcohol dosage (including assessment of a threshold effect) and alcohol-related problems as risk factors for tuberculosis. In particular, meta-analyses of the doseresponse relationship are of special importance, as these effects have been long suspected and are observed for other infectious diseases $[4,5]$. In addition, the alcohol-attributable tuberculosis burden of disease can be re-estimated based on updated risk relations (RRs), which may guide decision makers towards prevention and treatment interventions.

As such, the objectives of the present study were two-fold. First, meta-analyses of alcohol use, alcohol dosage and alcohol-related problems as risk factors for tuberculosis incidence (including recurrence) were conducted based on cohort and case-control studies. Second, alcohol-attributable tuberculosis incidence and mortality rates in 2014 were estimated for the 196 member states of the World Health Organization (WHO).

\section{Methods}

\section{Search strategy and selection criteria}

Meta-analyses of study-level observational data were undertaken and reported according to the Preferred Reporting Items for Systematic Reviews and Meta-Analyses (PRIMSA) guidelines [6]. Electronic searches were carried out for relevant studies in the PubMed database, as well as in a comprehensive tuberculosis studies database housed at the Global TB Programme of the WHO. The search strategies for these databases were identical to those operationalised in the prior meta-analysis by LÖNNROTH et al. [3]. As the prior meta-analysis by LÖNNROTH et al. [3] included studies published up to 2007, the present search identified studies published between January 2007 and June 2016. In addition to the above-mentioned database searches, the reference lists of all included studies were reviewed and expert consultations (with K. Lönnroth and J. Rehm) were held to further identify relevant studies. No language restrictions were applied and grey literature was included, but authors were not contacted.

Relevant studies were initially screened based on titles and abstracts, followed by full-text assessments. Inclusion criteria consisted of 1) cohort or case-control study design, 2) reporting of RRs for alcohol consumption as a risk factor for tuberculosis (such as relative risks, hazard ratios or odds ratios), or provision of sufficient information for the calculation of RRs, and 3) inclusion of tuberculosis incidence, new or recurrent, as an end-point. Relevant studies were excluded if they were not based on the most recent alcohol consumption exposure prior to or at the time of tuberculosis incidence or recurrence diagnosis, as alcohol consumption has been shown to decrease thereafter [7]. For example, case-control studies were excluded that enquired about current alcohol consumption at the time of interview from prevalent tuberculosis cases, as were cohort studies of tuberculosis recurrence that were based on alcohol consumption measured at baseline (i.e. not after the first treatment episode). Relevant studies with unclear temporal relationships were included as part of sensitivity analyses. Further details on the search strategy and selection criteria are available in appendix A.

\section{Tuberculosis case definition}

Tuberculosis was defined based on the International Statistical Classification of Diseases and Related Health Problems 10th Revision (ICD-10) codes A15-A19, as well as corresponding ICD-8 and ICD-9 codes.

\section{Alcohol consumption assessment}

Alcohol consumption was operationalised as alcohol use, alcohol dosage and alcohol-related problems. Alcohol use corresponded to measurements of lifetime or current use, as well as other qualitative and frequency-based measurements. Past alcohol use denoted measurements of former use (i.e. not current 
use). Alcohol dosage referred to measurements of ethanol (pure alcohol) intake in grammes per day. The midpoints of ethanol intake categories were calculated, whereas three-quarters of the second-highest category's range was added to the lower limit of open-ended categories. Conversion factors were applied when standard drinks were the unit of measurement. Depending on the country, one standard drink contains 8-14 g of ethanol [8]. Alcohol-related problems included measurements of alcohol use disorders, as well as physical health conditions related to chronic excessive alcohol consumption (e.g. alcoholic cirrhosis of the liver and alcoholic polyneuropathy). Detailed descriptions of the data extraction and quality assessment are available in appendix A.

\section{Meta-analyses}

The primary outcome was the RR for alcohol consumption as a risk factor for tuberculosis. Categorical meta-analyses were conducted for alcohol use (versus no alcohol use) and alcohol-related problems (versus no alcohol-related problems, including abstainers and drinkers) as risk factors for tuberculosis. Inverse variance weighted DerSimonian-Laird random effects models were used in these analyses to permit between-study heterogeneity [9]. The Cochran's Q and $\mathrm{I}^{2}$ statistics were computed to quantify variation in the RRs due to between-study heterogeneity, whereas p-values from Egger's regression-based test were computed for the assessment of publication bias [10-12]. Apart from these analyses, subgroup meta-analyses were conducted based on stratifications by study design and control for confounding, as well as based on the tuberculosis burden and problem drinking in the studied country. The stratification pertaining to the tuberculosis burden was based on the number of tuberculosis cases, as detailed in the Global Tuberculosis Report 2015 [13], whereas the stratification pertaining to problem drinking was based on total per capita alcohol consumption among drinkers and patterns of drinking scores from the Global Status Report on Alcohol and Health 2014 [14].

Linear dose-response meta-analyses were carried out for alcohol dosage as a risk factor for tuberculosis, where different levels of ethanol intake in grammes per day were modelled in comparison with no alcohol use (current abstention). Studies required a minimum of three or more ethanol intake categories for inclusion in the alcohol dosage meta-analyses. These analyses involved linear two-stage meta-regression models as described elsewhere [15]. In addition, categorical dose-response meta-analyses were conducted to investigate a potential threshold effect, using the categories of $\leqslant 24 \mathrm{~g},>24$ to $\leqslant 60 \mathrm{~g}$ and $>60 \mathrm{~g}$ of ethanol intake per day (all versus no alcohol use). As before, publication bias was assessed by using Egger's regression-based test[14].

All meta-analyses were conducted using Stata Statistical Software Version 14.1.

\section{Burden of disease analyses}

The alcohol-attributable tuberculosis burden of disease included incidence and mortality, which were estimated by using alcohol-attributable fractions [16]. These fractions estimate the proportion of tuberculosis incidence and mortality that would not have occurred under the theoretical minimum exposure of lifetime alcohol abstention [17]. As part of these estimations, alcohol consumption status data from 2012 (the latest year for which data were available) were combined with per capita alcohol consumption data from 2014 [14, 18, 19]. Alcohol consumption among current drinkers was modelled by using a continuous Gamma distribution (see references $[16,19]$ for the exact formulae). Alcohol consumption data were subsequently combined with the RRs from the linear dose-response meta-analyses to generate the alcohol-attributable fractions. Uncertainty intervals were generated by using 40000 Monte Carlo-like draws of all lowest level parameters [20]. The alcohol-attributable fractions were then applied to the tuberculosis incidence and mortality data from 2014, as obtained from the publically available Global Tuberculosis Database [21]. The alcohol-attributable tuberculosis burden of disease was estimated for the 196 member states, organised into six regions of the WHO in 2014. Comparisons of changes between 2000 and 2014 were also performed for the 22 high tuberculosis burden countries (same definition as above) [13]. Although both crude numbers and crude rates were generated, only the latter are discussed throughout the results. Compliance with the Guidelines for Accurate and Transparent Health Estimates Reporting (GATHER) Statement was ensured [22]. All burden of disease analyses were conducted using R Software Version 3.3.1. Further details on the methodology of the burden of disease analyses are presented in appendix B.

\section{Ethics approval}

Research ethics committee review and approval were not required, as aggregate data were extracted from published studies.

\section{Results}

The search strategy yielded 1108 relevant studies after duplicates were excluded (see figure S1 in appendix D for the PRISMA flow chart and checklist supplement for the PRISMA checklist). Based on title and 
abstract screening, 1015 of these studies were excluded, whereas 93 underwent full-text assessments. After completion of the full-text assessments, 36 studies in three languages (English, Portuguese and Chinese) were included in the meta-analyses [7, 23-57]. Among the included studies, eight were cohort studies and 28 were case-control studies. There was representation from all WHO regions, with the exception of the Eastern Mediterranean Region, as well as representation from several high tuberculosis burden countries, including India, China, South Africa, Russia and Brazil. Detailed descriptions of the included studies are presented in tables S1 and S2 in appendix D. From these included studies, 15 were part of the meta-analyses of alcohol use, four were part of the meta-analyses of alcohol dosage and eight were part of the meta-analyses of alcohol-related problems.

The RRs from the categorical meta-analyses of alcohol use as a risk factor for tuberculosis are presented in table 1 (see also tables S3 and S4 in appendix D for the sex-specific RRs) [7, 23-45]. Alcohol use was associated with a $35 \%$ higher risk of tuberculosis compared to no alcohol use (RR 1.35, 95\% CI 1.09-1.68). In some of the included studies, the reference categories of the alcohol consumption exposure included alcohol users (e.g. alcohol use never or less than or equal to 2 days per week versus alcohol use more than 2 days per week). There was an additional increase in tuberculosis risk once such studies were included in the meta-analyses. Past alcohol use was not significantly associated with a higher risk of tuberculosis compared to no alcohol use (RR 1.52, 95\% CI 0.67-3.43) (table S5 in appendix D).

Regression coefficients from the linear dose-response meta-analyses of alcohol dosage as a risk factor for tuberculosis are presented in table $2[24,25,28,35,39]$. The tuberculosis risk rose as ethanol intake in grammes per day increased: RR at $25 \mathrm{~g}$ per day: $1.57,95 \% \mathrm{CI} 1.10-2.23$; RR at $50 \mathrm{~g}$ per day: $2.46,95 \% \mathrm{CI}$ 1.21-4.98; RR at $75 \mathrm{~g}$ per day; 3.85, 95\% CI 1.33-11.11; and RR at $100 \mathrm{~g}$ per day: $6.03,95 \%$ CI $1.47-24.81$. The RRs from the related categorical dose-response meta-analyses are presented in table S6 in appendix D. The RRs were non-significant for the $\leqslant 24$ and $>24$ to $\leqslant 60$ categories of ethanol intake in grammes per day, but became significant thereafter along with increases in magnitude. Ethanol intake of more than $60 \mathrm{~g}$

TABLE 1 Risk relations (RRs) from categorical meta-analyses of alcohol use (versus no alcohol use) as a risk factor for tuberculosis

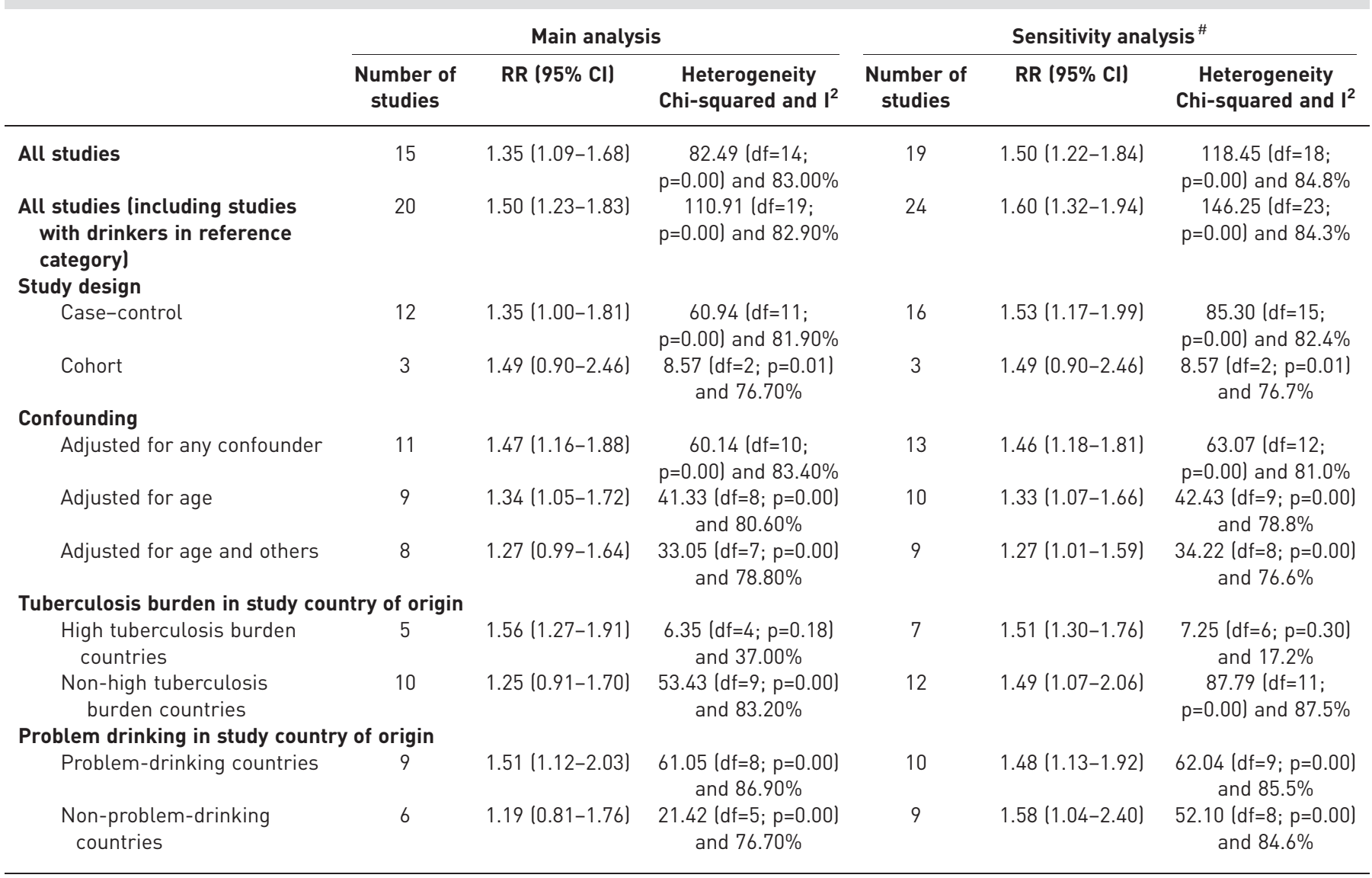

df: degrees of freedom. ${ }^{\#}$ : includes studies with unclear temporal relationships between exposure and outcome. 
TABLE 2 Regression coefficients from the linear dose-response meta-analyses of alcohol dosage in grammes per day of ethanol (versus no alcohol use) as a risk factor for tuberculosis

\begin{tabular}{|c|c|c|c|}
\hline \multicolumn{2}{|c|}{ Main analysis } & \multicolumn{2}{|c|}{ Sensitivity analysis ${ }^{\#}$} \\
\hline Number of studies & Beta $(95 \%$ CI) & Number of studies & Beta $(95 \% \mathrm{CI})$ \\
\hline 4 & $1.02(1.00-1.03)$ & 5 & $1.03(1.01-1.05)$ \\
\hline
\end{tabular}

per day was associated with a $68 \%$ higher risk of tuberculosis compared to no alcohol use (RR $1.68,95 \%$ CI 1.22-2.31).

Alcohol-related problems were associated with greater than a three-fold higher risk of tuberculosis compared to no alcohol-related problems (RR 3.33, 95\% CI 2.14-5.19) [46-57]. All RRs from the categorical meta-analyses of alcohol-related problems as a risk factor for tuberculosis are presented in table 3 .

As indicated by the Cochran's $\mathrm{Q}$ and $\mathrm{I}^{2}$ statistics, substantial between-study heterogeneity was detected. The subgroup meta-analyses based on the specified stratifications did not lead to extensive reductions in between-study heterogeneity, although there were a few exceptions. All p-values obtained from the Egger's regression-based test for publication bias were non-significant. Inclusion of studies that did not provide sufficient information for the ascertainment of alcohol consumption exposure timing did not lead to substantial changes in the overall pattern of findings. For a further discussion of the results pertaining to between-study heterogeneity, publication bias and sensitivity analyses, refer to appendix C.

Based on the RRs from the linear dose-response meta-analyses, alcohol consumption was estimated to have caused 1587449 incident cases of tuberculosis (95\% CI 1420559-2939075) globally in 2014, corresponding to 22.02 cases per 100000 people (95\% CI 19.70-40.77) (see also the GATHER checklist in the checklist

TABLE 3 Risk relations (RRs) from categorical meta-analyses of alcohol-related problems (versus no alcohol-related problems) as a risk factor for tuberculosis

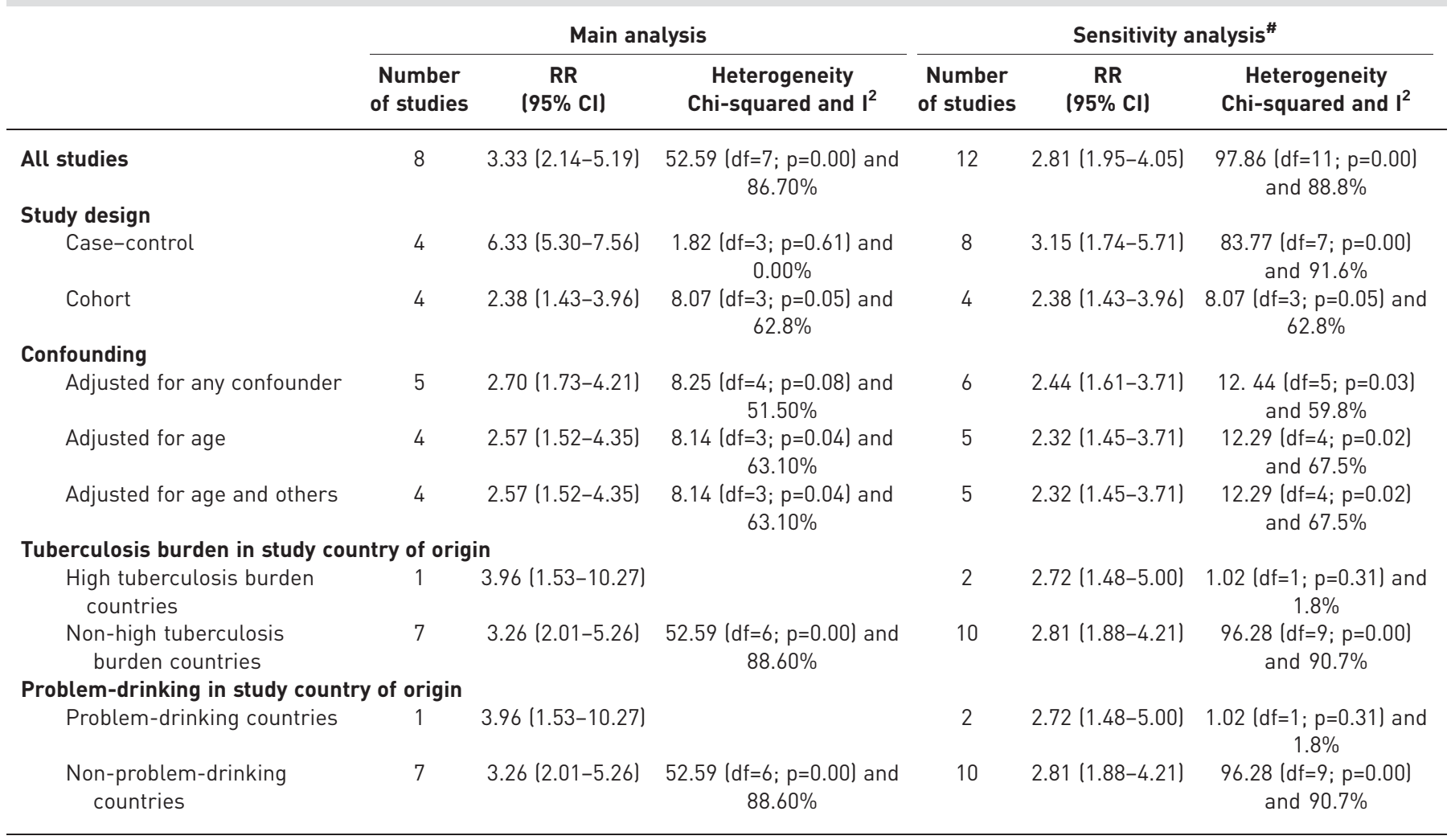

df: degrees of freedom. ${ }^{\#}$ : includes studies with unclear temporal relationships between exposure and outcome. 
TABLE 4 Estimated tuberculosis incidence and mortality (numbers and rates per 100000 people) attributable to alcohol consumption by World Health Organization regions in 2014

\begin{tabular}{|c|c|c|c|c|}
\hline \multirow[t]{2}{*}{ World Health Organization region } & \multicolumn{2}{|c|}{ Incidence } & \multicolumn{2}{|c|}{ Mortality } \\
\hline & Cases $(95 \% \mathrm{CI})$ & $\begin{array}{c}\text { Cases per } 100000 \\
\text { people }(95 \% \mathrm{CI})\end{array}$ & Deaths $(95 \% \mathrm{CI})$ & $\begin{array}{c}\text { Deaths per } 100000 \\
\text { people }(95 \% \mathrm{CI})\end{array}$ \\
\hline European region & $119056(94005-173664)$ & $13.13(10.37-19.15)$ & 12786 (9435-19863) & $1.41(1.04-2.19)$ \\
\hline African region & 507895 (394 259-835170) & $53.33(41.39-87.69)$ & 81774 (54698-157498) & $8.59(5.74-16.54)$ \\
\hline Western Pacific region & 402702 (242 688-698586) & 21.93 (13.22-38.05) & $21973(14622-35291)$ & $1.20(0.80-1.92)$ \\
\hline
\end{tabular}

supplement). The regional alcohol-attributable tuberculosis incidence estimates are presented in table 4, whereas the country-level estimates are displayed in figure 1 (see also table S7 in appendix D for the data). At the regional level, alcohol-attributable tuberculosis incidence was the highest in the African Region, followed by the South-East Asia Region and it was the lowest in the Eastern Mediterranean Region. Countries that featured alcohol-attributable tuberculosis incidence more than five times higher than the global average all belonged to the African Region: Swaziland, Gabon, Namibia, Lesotho and South Africa.

Alcohol consumption was also estimated to have caused 169721 tuberculosis deaths (95\% CI $148111-$ 345658 ) globally in 2014 (2.35 deaths per 100000 people, 95\% CI 2.05-4.79). The regional- and country-level estimates of alcohol-attributable tuberculosis mortality are presented in table 4 and figure 2, respectively (see also table S8 in appendix D for the data). The African Region featured the highest alcohol-attributable tuberculosis mortality, followed by the South-East Asia Region, whereas the lowest impacts were evident in the Eastern Mediterranean Region. Countries exhibiting alcohol-attributable tuberculosis mortality that was more than five times higher than the global average included Liberia, Angola, South Africa, Lesotho, Gabon, Namibia and Nigeria; all countries belonging to the African Region.

Changes in alcohol-attributable tuberculosis incidence and mortality between 2000 and 2014 among the 22 high tuberculosis burden countries are displayed in figures 3 and 4, respectively (see also tables S9 and S10 in appendix D for the data). Alcohol-attributable tuberculosis incidence increased in most high tuberculosis burden countries during the examined time period, with a mean percentage increase of $50 \%$

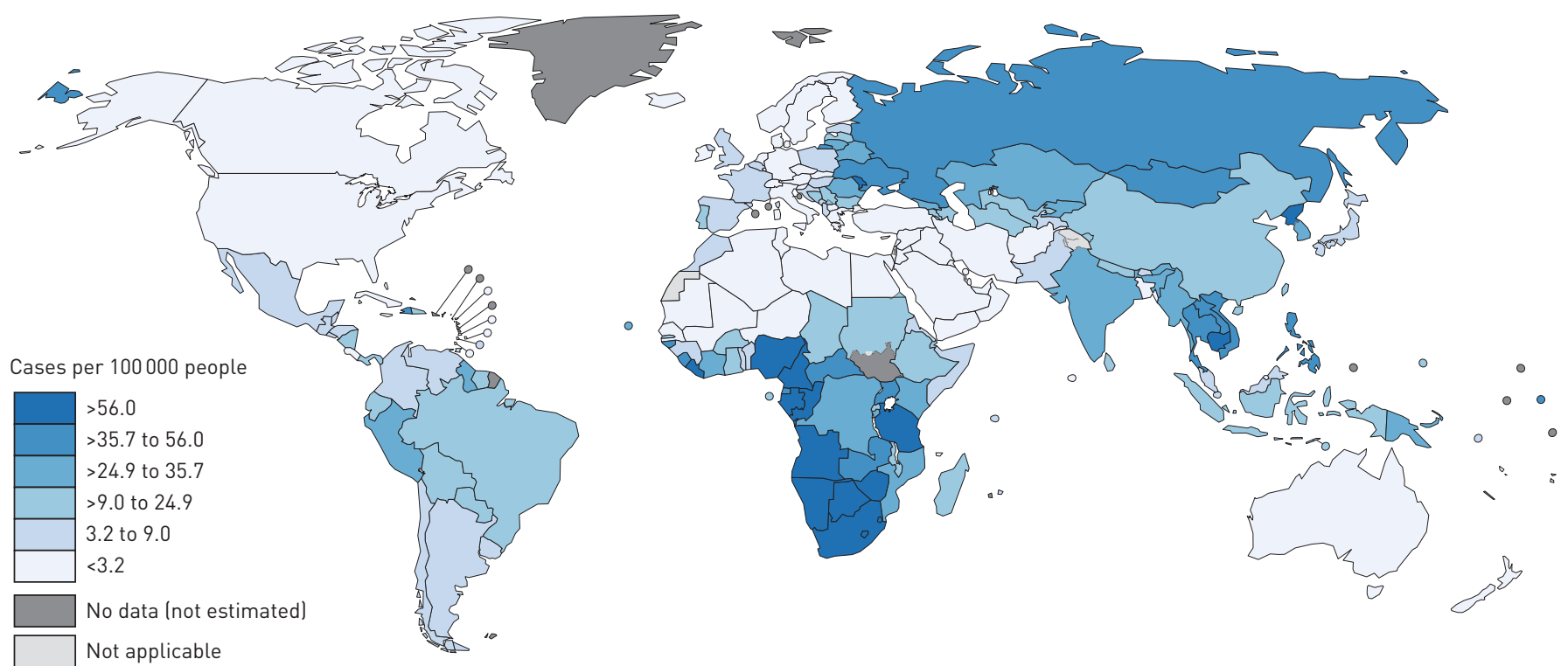

FIGURE 1 Estimated tuberculosis incidence rates per 100000 people attributable to alcohol consumption by countries in 2014 . The boundaries and names shown and the designations used on this map do not imply the expression of any opinion whatsoeveron the part of the World Health Organization concerning the legal status of any country territory city or area or of its authoritiesor concerning the delimitation of its frontiers or boundaries. Dotted and dashed lines on maps represent approximate border linesfor which there may not yet be full agreement. 

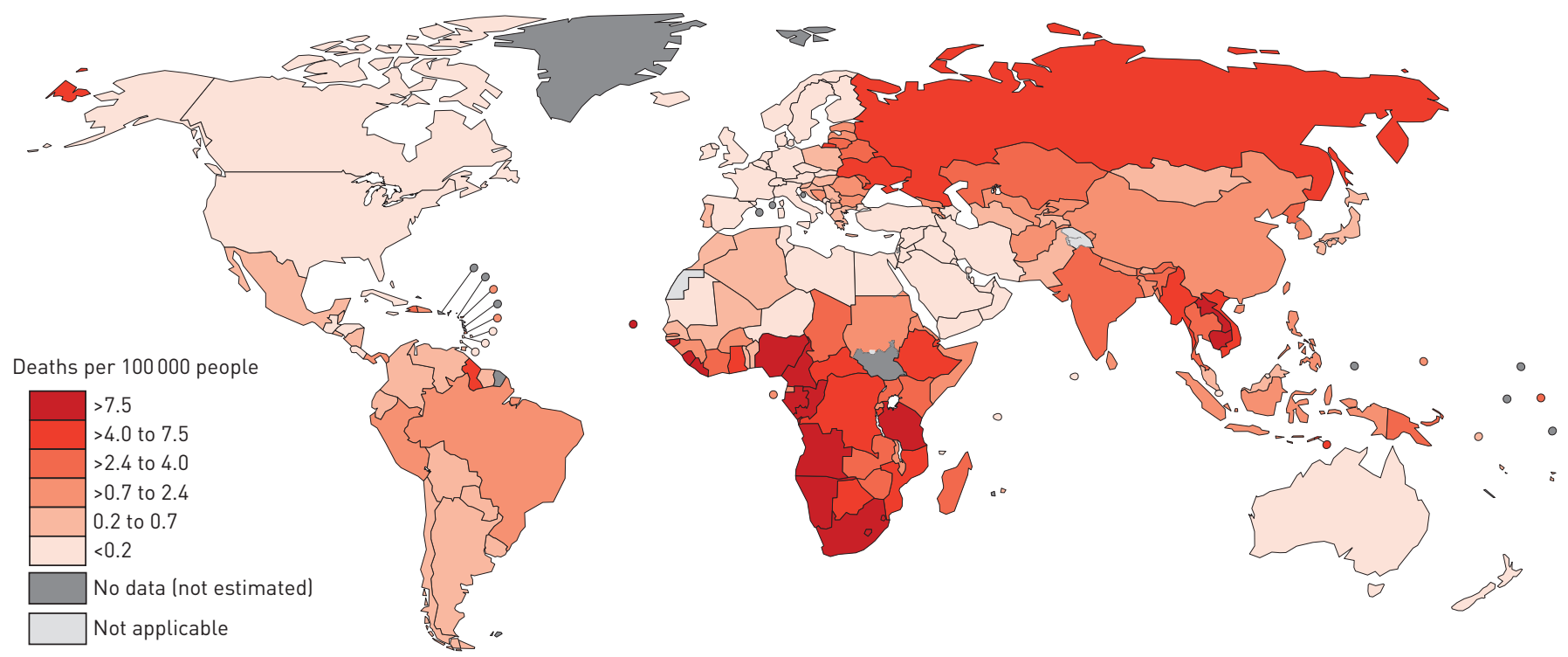

FIGURE 2 Estimated tuberculosis mortality rates per 100000 people attributable to alcohol consumption by countries in 2014 . The boundaries and names shown and the designations used on this map do not imply the expression of any opinion whatsoever on the part of the World Health Organization concerning the legal status of any country territory city or area or of its authorities or concerning the delimitation of its frontiers or boundaries. Dotted and dashed lines on maps represent approximate border lines for which there may not yet be full agreement.

(range 1-173\%). The most notable increases were observed in Myanmar, Vietnam and South Africa; all three countries recorded percentage increases of over 100\%. However, alcohol-attributable tuberculosis incidence decreased in Brazil, United Republic of Tanzania, Thailand, Russian Federation and Uganda (mean percentage decrease $13 \%$, range $1-43 \%$ ), with the greatest decreases observed in the latter two countries.

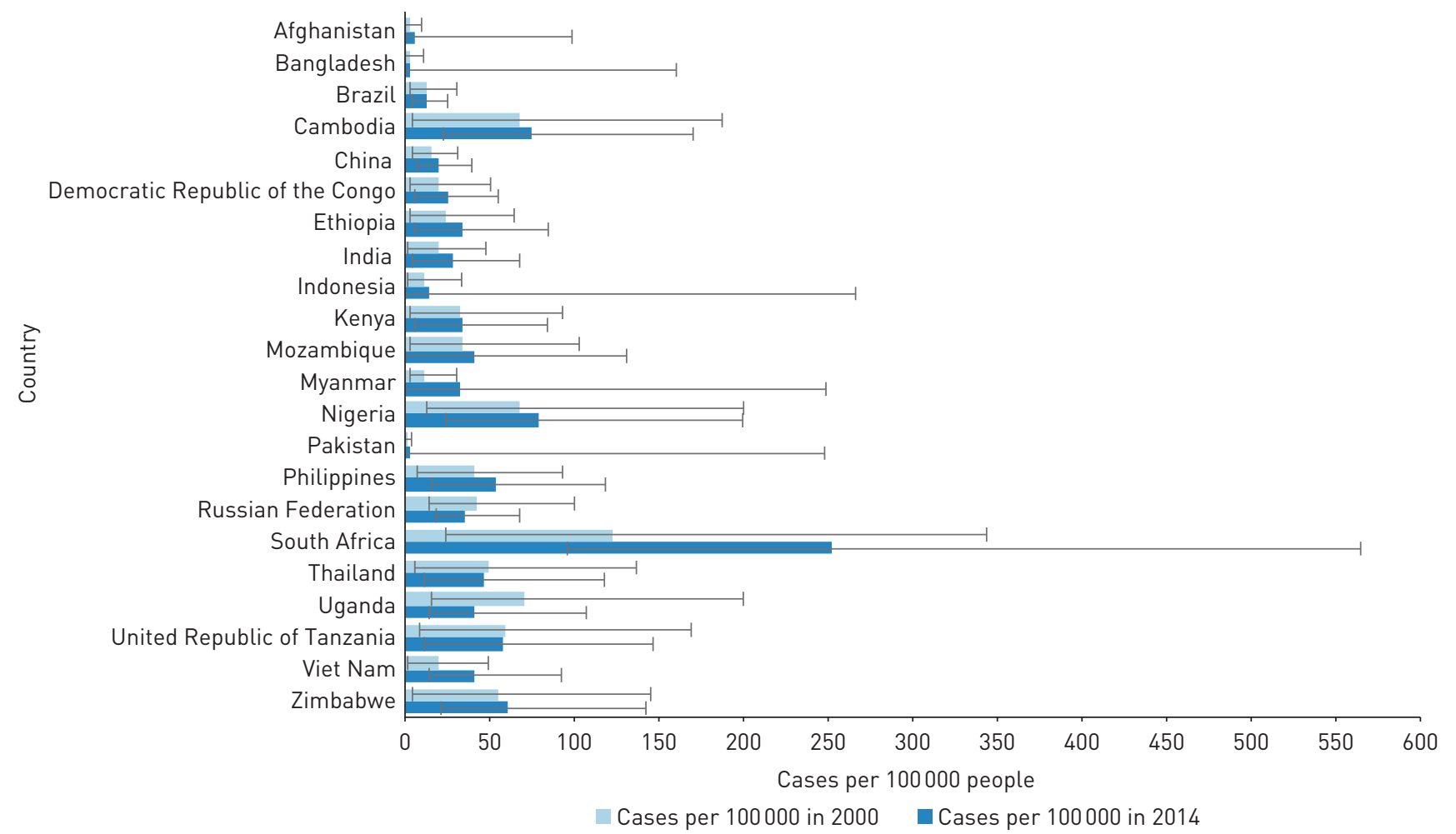

FIGURE 3 Estimated tuberculosis incidence rates per 100000 people attributable to alcohol consumption in high-tuberculosis burden countries for 2000 and 2014. 


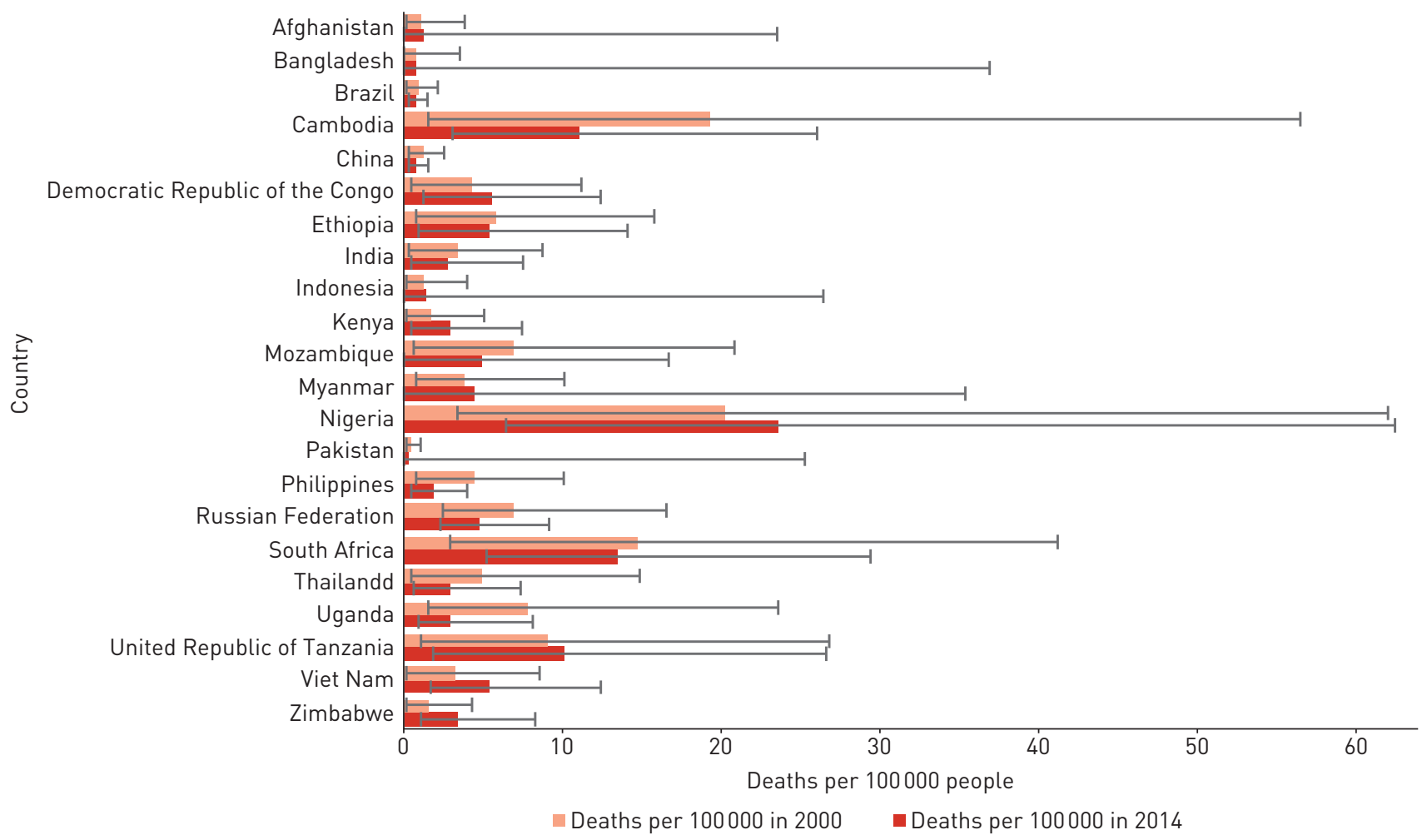

FIGURE 4 Estimated tuberculosis mortality rates per 100000 people attributable to alcohol consumption in high-tuberculosis burden countries for 2000 and 2014.

Alcohol-attributable tuberculosis mortality decreased in most high-tuberculosis-burden countries between 2000 and 2014, with a mean percentage decrease of 32\% (range 6-62\%). These decreases were particularly pronounced in Philippines and Uganda, where percentage decreases exceeded 50\%. Despite these decreases, alcohol-attributable tuberculosis mortality increased in 10 other countries (mean percentage increase 35\%, range $7-121 \%$ ), with percentage increases surpassing 50\% in Kenya, Vietnam and Zimbabwe.

\section{Discussion \\ Summary of findings}

Alcohol use, alcohol dosage and alcohol-related problems were examined as risk factors for tuberculosis through meta-analyses of cohort and case-control studies. All three classifications were associated with an increased risk of tuberculosis. As problems are associated with higher consumption [58], the dose-response meta-analyses suggest higher attributable tuberculosis burden of disease at the highest levels of alcohol consumption. Based on meta-analyses of alcohol dosage, the alcohol-attributable tuberculosis burden of disease was also estimated. Alcohol consumption was a major contributor to the tuberculosis burden of disease, with the most severe impacts estimated for the African Region.

\section{Strengths and limitations}

There are some notable strengths of the present study that deserve mention. Separate in-depth meta-analyses were conducted of alcohol use, alcohol dosage and alcohol-related problems as risk factors for tuberculosis. In particular, meta-analyses of alcohol dosage represent an important extension of prior research, as dose-response effects had been long suspected and were observed for other infectious diseases [4]. Apart from the in-depth meta-analyses, special attention was paid to the alcohol consumption exposure timing in the study selection, which diminished the risk of biasing the relationships. In addition to the meta-analyses, the alcohol-attributable tuberculosis burden of disease was estimated based on the updated RRs.

Despite the strengths of the present study, there are some limitations of the meta-analyses that should be considered. To be consistent with the meta-analysis conducted by LöNNROTH et al. [3], the database searches were limited to the two databases specified, which may have missed other relevant studies. A distinction between drug-susceptible tuberculosis and multidrug-resistant tuberculosis was not made, as alcohol consumption was considered to influence the risk in a similar manner. There is nonetheless some 
evidence to suggest that alcohol consumption impacts tuberculosis treatment compliance, leading to subsequent acquired drug resistance [59]. Several measurements of alcohol consumption in the included studies were susceptible to social desirability and recall biases (e.g. measurements from self-reported questionnaires), as well as misclassification bias (e.g. measurements from health administrative databases). Measurements of alcohol consumption in the included cohort studies did not account for changes over time, whereas not all measurements of alcohol-related problems were based on standardised diagnostic criteria. Additionally, there were a number of included studies that ascertained tuberculosis diagnosis from health administrative databases. These limitations may also have resulted also in misclassification bias. Apart from the limitations concerning measurements, substantial between-study heterogeneity was detected, which persisted in the subgroup meta-analyses. This can be attributed to differences between the included studies, such as sources of recruitment of participants, types of participants, definitions of alcohol consumption and definitions of tuberculosis. The low number of included studies also resulted in some underpowered subgroup meta-analyses, and precluded the quantification of sex-specific RRs in meta-analyses of alcohol dosage and alcohol-related problems.

There are also limitations of the burden of disease analyses. Alcohol consumption data were based on surveys and government statistics, with the former susceptible to issues of coverage and social desirability and recall biases. Dynamic modelling was not operationalised in these analyses, which precluded determination of the secondary effects of tuberculosis transmission from alcohol users to others. Additionally, the impact on mortality resulting from delayed diagnosis or treatment incompliance due to alcohol consumption was not considered. Based on these modelling decisions, the alcohol-attributable fractions should be considered conservative.

\section{Comparison with other studies}

In a previous meta-analysis related to the topic, LÖNNROTH et al. [3] showed that alcohol consumption of more than $40 \mathrm{~g}$ of ethanol per day or a diagnosis of an alcohol use disorder was associated with a nearly three-fold higher risk of tuberculosis (RR 2.94, 95\% CI 1.89-4.59) compared with no alcohol consumption or alcohol consumption below the specified thresholds. Although not directly comparable, the findings of LÖNNROTH et al. [3] are consistent with the RRs from the present meta-analyses of alcohol dosage and alcohol-related problems. Furthermore, the threshold effect of alcohol consumption was investigated in a sex-stratified case-control study [60]. The risk of tuberculosis was nonsignificant for males with an ethanol intake of less than $38 \mathrm{~g}$ per day, but significantly increased four-fold thereafter [60]. Alcohol consumption was not associated with tuberculosis for females [60]. The threshold effect observed in the case-control study was slightly lower than that estimated in the present meta-analyses (38 versus $60 \mathrm{~g}$ of ethanol intake per day) [60]. As such, further confirmation of the threshold effect is needed, especially as the category of $>24$ to $\leqslant 60$ g of ethanol intake per day included only one study in the present meta-analyses.

The alcohol-attributable tuberculosis burden of disease on the other hand has not been frequently estimated [61]. In the most comparable iteration of the Global Burden of Disease Study, alcohol-attributable tuberculosis mortality totalled 155000 deaths (86000-200000) in 2013 [62]. This is largely consistent with the mortality estimates generated in the present study. The minor variation in estimates can be attributed to the modelling time frame, as well as to the choice of RRs and consequential modelling strategy, as the Global Burden of Disease Study operationalised the RRs computed by LÖNNROTH et al. [3].

\section{Explanations and implications}

The relationship between alcohol consumption and tuberculosis can be explained by two causal pathways $[3,4]$. First, alcohol consumption impairs the immune system, which increases susceptibility to tuberculosis infection, as well as to reactivation of latent tuberculosis [3, 4]. The ability of alveolar macrophages to respond to newly introduced pathogens is compromised by alcohol consumption [63]; under normal circumstances these macrophages eliminate most mycobacteria [64]. Specifically, mobilisation and adherence of macrophages is suppressed and phagocytosis of mycobacteria and superoxide production are impacted [65-69]. In addition, monocytes' production of cytokines that regulate inflammation is limited by alcohol consumption [70,71]. The macrophages' ability to respond to these cytokines and present mycobacterial antigens to lymphocytes is also reduced, and antigen-specific T-cell activation is impaired [69-71]. As a result of these changes, the immune system's ability to respond to new and dormant mycobacteria is severely diminished [72]. Apart from direct influences on the immune system, alcohol consumption is associated with malnutrition, liver disease and social drift, all of which impact the maintenance of innate and acquired immune systems [72-76]. Second, alcohol consumption leads to presence in social environments that facilitate the spread of tuberculosis infection [3, 4]. Examples of such environments include bars, shelters, prisons and social institutions, which have been implicated in molecular-epidemiological studies [77-79]. 
Regarding the burden of disease, alcohol consumption was responsible for $17 \%$ of incident cases and $15 \%$ of deaths due to tuberculosis. Alcohol-attributable tuberculosis incidence increased with tuberculosis incidence, as expected; however, an important exception was the Eastern Mediterranean Region, where tuberculosis incidence was the third highest [21], but alcohol-attributable tuberculosis incidence was the lowest, given the low alcohol consumption [14, 18]. This trend was less apparent in the case of alcohol-attributable tuberculosis mortality. The European Region featured the third highest alcohol-attributable tuberculosis mortality, despite having one of the lowest tuberculosis mortality rates [21]; this was driven by high alcohol consumption, as determined by both the proportion of current drinkers and total per capita alcohol consumption [14, 18]. Concerning changes between 2000 and 2014, the global tuberculosis burden of disease has been declining for almost the past two decades. There are still important differences in the extent of these declines, as the reductions in tuberculosis mortality compared to incidence have been significantly larger (38\% versus 18\% cumulative rate reductions between 2000 and 2014, respectively) [21]. Nonetheless, the tuberculosis burden of disease decreased during the examined time period in the overwhelming majority of the high tuberculosis burden countries, with a few exceptions from the African Region [21]. It therefore follows that increases in the alcohol-attributable tuberculosis burden of disease in these countries were due primarily to increases in alcohol consumption.

The global strategy for tuberculosis control has evolved to incorporate a broader preventative focus [80]. This shift has been a consequence of lower than expected reductions in the tuberculosis burden of disease, especially its incidence $[81,82]$. Findings from the present study emphasise one important component of the current global strategy for tuberculosis control: the necessity of interventions to prevent heavy alcohol consumption and alcohol-related problems, as well as to address shared social determinants of health [80]. This component is based on the rationale that targeting risk factors and social determinants of health will supplement cutting transmission chains, which will further reduce the tuberculosis burden of disease [81, 83]. Indeed, it is relevant to a number of countries that have observed increases in alcohol consumption, including high tuberculosis burden countries. To that end, interventions can be implemented to reduce the harms of alcohol consumption, including regulation of the environment in which alcohol is marketed, especially its price and availability, as well as individually directed interventions for those with at-risk levels of alcohol consumption [84]. However, targeting shared social determinants of health will produce greater public health benefits, which can be addressed by the frameworks for action developed by the Commission on Social Determinants of Health [85].

In addition to tuberculosis control, findings from the present study emphasise that addressing heavy alcohol consumption and alcohol-related problems are of relevance for eventual tuberculosis elimination, particularly in low-incidence settings [86]. This is because as the incidence of tuberculosis decreases, it becomes more and more concentrated among vulnerable risk groups, including those with heavy alcohol consumption and alcohol-related problems [86]. As such, on top of the prevention interventions described above, training and awareness initiatives for relevant healthcare professionals should be considered, especially as they relate to assisting with access to and navigation of tuberculosis care [86].

\section{Conclusion}

Alcohol use, alcohol dosage and alcohol-related problems were all associated with an increased risk of tuberculosis in meta-analyses of cohort and case-control studies. Based on the updated meta-analyses, alcohol consumption was a major contributor to the tuberculosis burden of disease, particularly in the African Region. As such, alcohol control policies have an instrumental role to play within the global strategy for tuberculosis control.

\section{Acknowledgements}

The authors wish to thank Maximilien Rehm (Ritsumeikan University, Kyoto, Japan), Kwun (Bryan) Hung Chan (The Chinese University of Hong Kong, Hong Kong, China) and Joyce Cheng (Centre for Addiction and Mental Health, Toronto, Canada) for assisting with the translations of non-English studies, as well as Michelle Tortolo (Centre for Addiction and Mental Health, Toronto, Canada) for referencing the manuscript.

The study had no funding source. It was carried out as part of the WHO Collaborating Centre on Mental Health and Addiction, which has been responsible for estimating the alcohol-attributable burden of disease for the Global Status Reports on Alcohol and Health [14, 18].

\section{References}

1 World Health Organization. Global Tuberculosis Report. World Health Organization, Geneva, Switzerland, 2016.

2 Institute for Health Metrics and Evaluation. GBD Compare. 2015. Date last acessed: July 19, 2016. http://vizhub. healthdata.org/gbd-compare

3 Lönnroth K, Williams B, Stadlin S, et al. Alcohol use as a risk factor for tuberculosis - a systematic review. BMC Public Health 2008; 8: 289.

4 Rehm J, Samokhvalov AV, Neuman MG, et al. The association between alcohol use, alcohol use disorders and tuberculosis (TB). A systematic review. BMC Public Health 2009; 9: 450. 
5 Rehm J, Baliunas D, Borges GL, et al. The relation between different dimensions of alcohol consumption and burden of disease - an overview. Addiction 2010; 105: 817-843.

6 Moher D, Liberati A, Tetzlaff J, et al. Preferred reporting items for systematic reviews and meta-analyses: the PRISMA statement. PLoS Med 2009; 6: e1000097.

7 Tocque $\mathrm{K}$, Bellis M, Beeching NJ, et al. A case-control study of lifestyle risk factors associated with tuberculosis in Liverpool, North-West England. Eur Respir J 2001; 18: 959-964.

8 World Health Organization. International Guide for Monitoring Alcohol Consumption and Related Harm. Geneva, World Health Organization, 2000.

9 DerSimonian R, Laird N. Meta-analysis in clinical trials. Control Clin Trials 1986; 7: 177-188.

10 Cochran WG. The combination of estimates from different experiments. Biometrics 1954; 10: 101-129.

11 Higgins JP, Thompson SG. Quantifying heterogeneity in a meta-analysis. Stat Med 2002; 21: 1539-1558.

12 Egger M, Smith GD, Schneider M, et al. Bias in meta-analysis detected by a simple, graphical test. BMJ 1997; 315: 629-634.

13 World Health Organization. Global Tuberculosis Report. Geneva, World Health Organization, 2015.

14 World Health Organization. Global Status Report on Alcohol and Health. Geneva, World Health Organization, 2014.

15 Orsini NBR, Greenland S. Generalized least squares for trend estimation of summarized dose-response data. Stata J 2006; 6: 40-57.

16 Kehoe T, Gmel G Jr, Shield K, et al. Determining the best population-level alcohol consumption model and its impact on estimates of alcohol-attributable harms. Popul Health Metr 2012; 10: 6.

17 Murray CJL, Lopez A. On the comparable quantification of health risks: lessons from the global burden of disease study. Epidemiology 1999; 10: 594-605.

18 World Health Organization. Global status report on alcohol. Geneva, World Health Organization, 2004.

19 Rehm J, Kehoe T, Gmel G, et al. Statistical modeling of volume of alcohol exposure for epidemiological studies of population health: the example of the US. Popul Health Metr 2010; 8: 3.

20 Gmel GJ, Shield KD, Frick H, et al. Estimating uncertainty of alcohol-attributable fractions for infectious and chronic diseases. BMC Med Res Methodol 2011; 11: 48.

21 World Health Organization. WHO TB burden estimates. 2016. Date last accessed: October 5, 2016. www.who.int/ tb/country/data/download/en/

22 Stevens GA, Alkema L, Black RE, et al. Guidelines for accurate and transparent health estimates reporting: the GATHER statement. Lancet 2016; 388: e19-e23.

23 Gajalakshmi V, Peto R. Smoking, drinking and incident tuberculosis in rural India: population-based case-control study. Int J Epidemiol 2009; 38: 1018-1025.

24 Jee SH, Golub JE, Jo J, et al. Smoking and risk of tuberculosis incidence, mortality, and recurrence in South Korean men and women. Am J Epidemiol 2009; 170: 1478-1485.

25 Amoakwa K, Martinson NA, Moulton LH, et al. Risk factors for developing active tuberculosis after the treatment of latent tuberculosis in adults infected with human immunodeficiency virus. Open Forum Infect Dis 2015; 2: ofu120.

26 Hemilä H, Kaprio J, Pietinen P, et al. Vitamin C and other compounds in vitamin C rich foods in relation to risk of tuberculosis in male smokers. Am J Epidemiol 1999; 150: 632-641.

27 Rosenman K, Hall N. Occupational risk factors for developing tuberculosis. Am J Ind Med 1996; 30: 148-154.

28 Buskin SE, Gale JL, Weiss NS, et al. Tuberculosis risk factors in adults in King County, Washington, 1988 through 1990. Am J Public Health 1994; 84: 1750-1756.

29 Chen M, Deng J, Li W, et al. Impact of tea drinking upon tuberculosis: a neglected issue. BMC Public Health 2015; 15: 515.

30 Kolappan C, Subramani R. Association between biomass fuel and pulmonary tuberculosis: a nested case-control study. Thorax 2009; 64: 705-708.

31 Ladefoged K, Rendal T, Skifte T, et al. Risk factors for tuberculosis in Greenland: case-control study. Int J Tuberc Lung Dis 2011; 15: 44-49.

32 Coker R, McKee M, Atun R, et al. Risk factors for pulmonary tuberculosis in Russia: case-control study. BMJ 2006; 332: 85-87.

33 Shetty N, Shemko M, Vaz M, et al. An epidemiological evaluation of risk factors for tuberculosis in South India: a matched case control study. Int J Tuberc Lung Dis 2006; 10: 80-86.

34 Crampin AC, Glynn JR, Floyd S, et al. Tuberculosis and gender: exploring the patterns in a case control study in Malawi. Int J Tuberc Lung Dis 2004; 8: 194-203.

35 Brown KE, Campbell AH. Tobacco, alcohol and tuberculosis. Br J Dis Chest 1961; 55: 150-158.

36 Lewis D, Chamberlain J. Alcohol consumption and smoking habits in male patients with pulmonary tuberculosis. Br J Prev Soc Med 1963; 17: 149-152.

37 Tekkel M, Rahu M, Loit HM, et al. Risk factors for pulmonary tuberculosis in Estonia. Int J Tuberc Lung Dis 2002; 6: 887-894.

38 Lienhardt C, Fielding K, Sillah JS, et al. Investigation of the risk factors for tuberculosis: a case-control study in three countries in West Africa. Int J Epidemiol 2005; 34: 914-923.

39 Spletter ED. Association between active pulmonary tuberculosis, tobacco smoke and alcohol consumption: a case control study (Academic thesis). Midwestern University, Glendale, USA, 2000.

40 Dong B, Zhou Y. Smoking and alcohol consumption as risk factors of pulmonary tuberculosis in Chengdu: a matched case-control study. Hua Xi Yi Ke Da Xue Xue Bao 2001; 32: 104-106.

41 Baker MA, Lin HH, Chang HY, et al. The risk of tuberculosis disease among persons with diabetes mellitus: a prospective cohort study. Clin Infect Dis 2012; 54: 818-825.

42 Hill PC, Jackson-Sillah D, Donkor SA, et al. Risk factors for pulmonary tuberculosis: a clinic-based case control study in The Gambia. BMC Public Health 2006; 6: 156

43 Pokhrel AK, Bates MM, Verma SC, et al. Tuberculosis and indoor biomass and kerosene use in Nepal: a case-control study. Environ Health Perspect 2010; 118: 558-564.

44 Ruffino-Netto A, Caron-Ruffino M. Interação de fatores riscos em tuberculose. Rev Saude Publica 1979; 13: $119-122$ 
45 Souza de Lima D, Morishi Ogusku M, Porto Dos Santos M, et al. Alleles of HLA-DRB1* 04 Associated with Pulmonary Tuberculosis in Amazon Brazilian Population. PloS One 2016; 11: e0147543.

46 Kim S, Crittenden K. Risk factors for tuberculosis among inmates: a retrospective analysis. Public Health Nurs 2005; 22: 108-118.

47 Mori MA, Leonardson G, Welty TK. The benefits of isoniazid chemoprophylaxis and risk factors for tuberculosis among Oglala Sioux Indians. Ann Intern Med 1992; 152: 547-550.

48 Inghammar M, Löfdahl CG, Winqvist $\mathrm{N}$, et al. Impaired pulmonary function and the risk of tuberculosis: a population-based cohort study. Eur Respir J 2011; 37: 1285-1287.

49 Lin YT, Wu PH, Lin CY, et al. Cirrhosis as a risk factor for tuberculosis infection - a nationwide longitudinal study in Taiwan. Am J Epidemiol 2014; 180: 103-110.

50 Hsu WH, Kuo CH, Wang SS, et al. Acid suppressive agents and risk of Mycobacterium tuberculosis: case-control study. BMC Gastroenterol 2014; 14: 91.

51 Leegaard A, Riis A, Kornum JB, et al. Diabetes, glycemic control, and risk of tuberculosis: a population-based case-control study. Diabetes Care 2011; 34: 2530-2535.

52 Mahuad C, Bozza V, Pezzotto SM, et al. Impaired immune responses in tuberculosis patients are related to weight loss that coexists with an immunoendocrine imbalance. Neuroimmunomodulation 2007; 14: $193-199$.

53 Moran-Mendoza O, Marion SA, Elwood K, et al. Risk factors for developing tuberculosis: a 12-year follow-up of contacts of tuberculosis cases. Int J Tuberc Lung Dis 2010; 14: 1112-1119.

54 Inghammar M, Ekbom A, Engström G, et al. COPD and the risk of tuberculosis-a population-based cohort study. PloS One 2010; 5: e10138.

55 Boccia D, Hargreaves J, De Stavola BL, et al. The association between household socioeconomic position and prevalent tuberculosis in Zambia: a case-control study. PloS One 2011; 6: e20824.

56 Oliveira HBD, Moreira Filho DDC. Recidivas em tuberculose e seus fatores de risco. Rev Panam Salud Pública 2000; 74: 232-241

57 Menezes AMB, Costa JDD, Gonçalves $\mathrm{H}$, et al. Incidência e fatores de risco para tuberculose em Pelotas, uma cidade do Sul do Brasil. Rev Bras Epidemiol 1998; 1: 50-60.

58 Rehm J, Anderson P, Gual A, The tangible common denominator of substance use disorders: a reply to commentaries to Rehm et al. (2013). Alcohol Alcohol 2014; 49: 118-122.

59 Simet SM, Sisson JH. Alcohol's effects on lung health and immunity. Alcohol Res 2015; 37: 199-208.

60 Francisco J, Oliveira O, Felgueiras Ó, et al. How much is too much alcohol in tuberculosis? Eur Respir J 2016; 49: 1601468.

61 Shield K, Samokhvalov A, Rehm J. Global burden of tuberculosis and lower respiratory infections attributable to alcohol consumption in 2004. Int J Alcohol Drug Res 2013; 2: 11-18.

62 Forouzanfar $\mathrm{MH}$, Alexander L, Anderson HR, et al. Global, regional, and national comparative risk assessment of 79 behavioural, environmental and occupational, and metabolic risks or clusters of risks in 188 countries, 1990 2013: a systematic analysis for the Global Burden of Disease Study 2013. Lancet 2015; 386: 2287-2323.

63 Joshi PC, Applewhite L, Ritzenthaler JD, et al. Chronic ethanol ingestion in rats decreases granulocyte-macrophage colony-stimulating factor receptor expression and downstream signaling in the alveolar macrophage. J Immunol 2005; 175: 6837-6845.

64 Dannenberg AM. Immune mechanisms in the pathogenesis of pulmonary tuberculosis. Rev Infect Dis 1989; 11: S369-S378.

65 Castro A, Lefkowitz DL, Lefkowitz SS. Effects of alcohol on murine macrophage function. Life Sci 1993; 52: 1585-1593.

66 Dorio RJ, Forman HJ. Ethanol inhibition of signal transduction in superoxide production by rat alveolar macrophages. A proposed mechanism for ethanol related pneumonia. Ann Clin Lab Sci 1988; 18: 190-194.

67 Rimland D, Hand WL. The effect of ethanol on adherence and phagocytosis by rabbit alveolar macrophages. J Lab Clin Med 1980; 95: 918-926.

68 Rimland K. Mechanisms of ethanol-induced defects of alveolar macrophage function. Alcohol Clin Exp Res 1983; 8: $73-76$

69 Bermudez L, Young LS. Ethanol augments intracellular survival of mycobacterium avium complex and impairs macrophage responses to cytokines. J Infect Dis 1991; 163: 1286-1292.

70 Crews FT, Bechara R, Brown LA, et al. Cytokines and alcohol. Alcohol Clin Exp Res 2006; 30: 720-730.

71 Gamble L, Mason CM, Nelson S. The effects of alcohol on immunity and bacterial infection in the lung. Med Mal Infect 2006; 36: 72-77.

72 Szabo G, Saha B. Alcohol's effect on host defense. Alcohol Res 2015; 37: 159-170.

73 Szabo G. Alcohol and susceptibility to tuberculosis. Alcohol Health Res World 1997; 21: 39-41.

74 Szabo G. Alcohol's contribution to compromised immunity. Alcohol Health Res World 1997; 21: 30-41.

75 Lieber CS. Biochemical and molecular basis of alcohol-induced injury to liver and other tissues. $N$ Engl J Med 1988; 319: 1639-1650.

76 Dunne FJ. Alcohol and immune system. A causative agent in altering host defence mechanisms. BMJ 1989; 289: $543-544$

77 Diel R, Schneider S, Meywald-Walter K, et al. Epidemiology of tuberculosis in Hamburg, Germany: long-term population-based analysis applying classical and molecular epidemiological techniques. J Clin Microbiol 2002; 4 : 532-539.

78 Zolnir-Dovc M, Poljak M, Erzen D, et al. Molecular epidemiology of tuberculosis in Slovenia: results of a one-year (2001) nation-wide study. Scand J Infect Dis 2003; 35: 863-868.

79 Classen $\mathrm{CN}$, Warren R, Richardson M, et al. Impact of social interactions in the community on the transmission of tuberculosis in a high incidence area. Thorax 1999; 54: 136-140.

80 World Health Organization. The End TB Strategy: Global Strategy and Targets for Tuberculosis Prevention, Care and Control After 2015. Geneva, World Health Organization, 2015.

81 Lönnroth K, Jaramillo E, Williams BG, et al. Drivers of tuberculosis epidemics: the role of risk factors and social determinants. Soc Sci Med 2009; 68: 2240-2246.

82 Uplekar M, Weil D, Lönnroth K, et al. WHO’s new End TB Strategy. Lancet 2015; 385: 1799-1801. 
83 Lönnroth K, Castro KG, Chakaya JM, et al. Tuberculosis control and elimination 2010-50: cure, care, and social development. Lancet 2010; 375: 1814-1829.

84 Anderson P, Chisholm D, Fuhr D. Effectiveness and cost-effectiveness of policies and programmes to reduce the harm caused by alcohol. Lancet 2009; 373: 2234-2246.

85 World Health Organization. Closing the gap in a generation: health equity through action on the social determinants of health. Commission on Social Determinants of Health Final Report. Geneva, World Health Organization, 2008.

86 Lönnroth K, Migliori GB, Abubakar I, et al. Towards tuberculosis elimination: an action framework for low-incidence countries. Eur Respir J 2015; 45: 928-952. 